\title{
Cost effectiveness and cost utility of preventing trachomatous visual impairment: lessons from 30 years of trachoma control in Burma
}

\author{
T G Evans, M K Ranson, Tun Aung Kyaw, Chit Ko Ko
}

\begin{abstract}
Aims/background-This paper reports on the findings of a cost and effectiveness study of the trachoma control programme (TCP) in Burma. The TCP began in 1964 employing non-surgical interventions (community education and mass treatment with topical antibiotics) and surgical correction of trichiasis.

Methods-Fixed and variable costs of the TCP are assessed over 30 years (1964-93) and apportioned to either surgical or nonsurgical interventions. The change in the prevalence of trachoma blindness during this period is used to calculate cases of visual impairment prevented by the TCP. The years of life saved because of premature mortality averted and from living in a handicapped state are added to yield a single measure of utility called handicap adjusted life years (HALYs).
\end{abstract}

Results-The cost effectiveness of the TCP is $\$ 54$ per case of visual impairment prevented: $\$ 193$ and $\$ 47$ for surgical and non-surgical interventions respectively. The cost utility of the TCP is \$4 per HALY averted: $\$ 10$ and $\$ 3$ for surgical and non-surgical interventions respectively. Results are highly sensitive to the 1965 prevalence of blindness, the choice of discount rate, and the effectiveness of both interventions.

Conclusions-Thirty years of trachoma control in Burma are associated with a remarkable decline in trachomatous blindness. Both surgical and non-surgical interventions are cost effective means of preventing trachomatous visual impairment. Discussion focuses on methodological limitations and implications for research and policy. (Br f Ophthalmol 1996;80:880-889)

Trachoma, from the Greek words for 'rough' and 'swelling', has been a major cause of blindness for centuries, occurring mainly among those who are socioeconomically disadvantaged, living in hot dry climates where access to adequate water supplies is often limited. Acute infection with the Gram negative bacterium Chlamydia trachomatis causing an inflammation of the conjunctiva mainly affects children, whereas the complications of trachoma infection, such as scarring of the conjunctiva, inward deviation of the eyelid (entropion) and eyelashes (trichiasis), and corneal opacification develop later in life. ${ }^{1}$ In many industrialised countries, blinding trachoma gradually disappeared as standards of living improved in the 19th and early 20th centuries. In less developed areas, however, trachomatous visual impairment remained a major public health problem, especially among the rural poor.

After the second world war, technical advances in medical and surgical therapies, along with the creation of United Nations agencies such as the World Health Organisation and UNICEF, ushered in an era of active disease control or eradication. Correspondingly, major 'vertical' national campaigns were mounted against trachoma employing newly developed tetracycline topical treatments for Chlamydia trachomatis in addition to dedicated personnel and infrastructure. In the late $1970 \mathrm{~s}$, with the emergence of primary health care, these disease specific vertical programmes became outmoded and were replaced by 'horizontal' initiatives integrated into the primary health care system. Accordingly, trachoma control interventions were conceived within the framework of the existing health care system, becoming a major component of primary eye care programmes.

In the past 30 years, a number of countries have documented impressive declines in the prevalence of trachoma blindness to the point that trachoma is no longer considered a public health problem. The relative contribution to this success of vertical and/or horizontal trachoma control programmes in addition to improvement in standards of living remains undetermined. Recognising that there are areas where trachomatous visual impairment continues to exact a heavy toll, an analysis of these successful control experiences may provide direction to trachoma programmes seeking to maximise effectiveness and a rationale for ministries of health to support trachoma control activities.

To this end, this paper compares the costs and benefits of two types of trachoma interventions employed over a 30 year period by the trachoma control programme (TCP) in Burma (see Fig 1) with the aim of deriving insights on vertical and horizontal approaches to trachoma control, in addition to identifying the optimal mix of interventions to prevent trachomatous visual impairment. We use the techniques of cost effectiveness analysis, in which outcomes are measured in units of health; and cost utility analysis, in which the social value of the outcomes is measured. To our knowledge, 
there are no previous studies which assess the cost effectiveness of trachomatous visual impairment prevention in Burma or elsewhere. It needs to be emphasised that the outcome measure of interest in this study is prevention of trachomatous visual impairment. Other outcomes of the trachoma interventions being compared, such as the treatment of trachoma infection, are acknowledged but are not considered in the analysis.

Following a brief discussion of trachoma and its control in Burma, the costs of organising and operating non-surgical and surgical interventions are calculated for three distinct phases of the programme. Drawing on studies of the prevalence of trachomatous blindness in Burma and of the efficacy of surgical prevention, the effectiveness and utility of nonsurgical and surgical interventions are derived and expressed as the number of cases of visual impairment prevented and handicap adjusted life years (HALYs) saved, respectively. HALYs are a composite indicator of health status which incorporate information on premature mortality and years of life lived with visual impairment. The cost effectiveness and cost utility of non-surgical and surgical interventions are calculated for each of the programme's three phases. A sensitivity analysis identifies the range of cost utility values obtained when single variables are altered from low to high through their ranges of uncertainty. Our discussion focuses on the implications of our findings for the evaluation and planning of similar programmes elsewhere.

\section{Trachoma control in Burma}

The socioeconomic and climatic conditions of central Burma, also known as the 'dry zone', make it a suitable milieu for high rates of trachoma infection and blindness as identified by reports in the early 1960s. ${ }^{2}$ Consequently, the Ministry of Health launched the trachoma control programme (TCP) in 1964, employing a staff of only 39 and covering 11 townships in two districts with an estimated population of 1.5 million. Today, more than 400 people are employed by the programme, which covers a

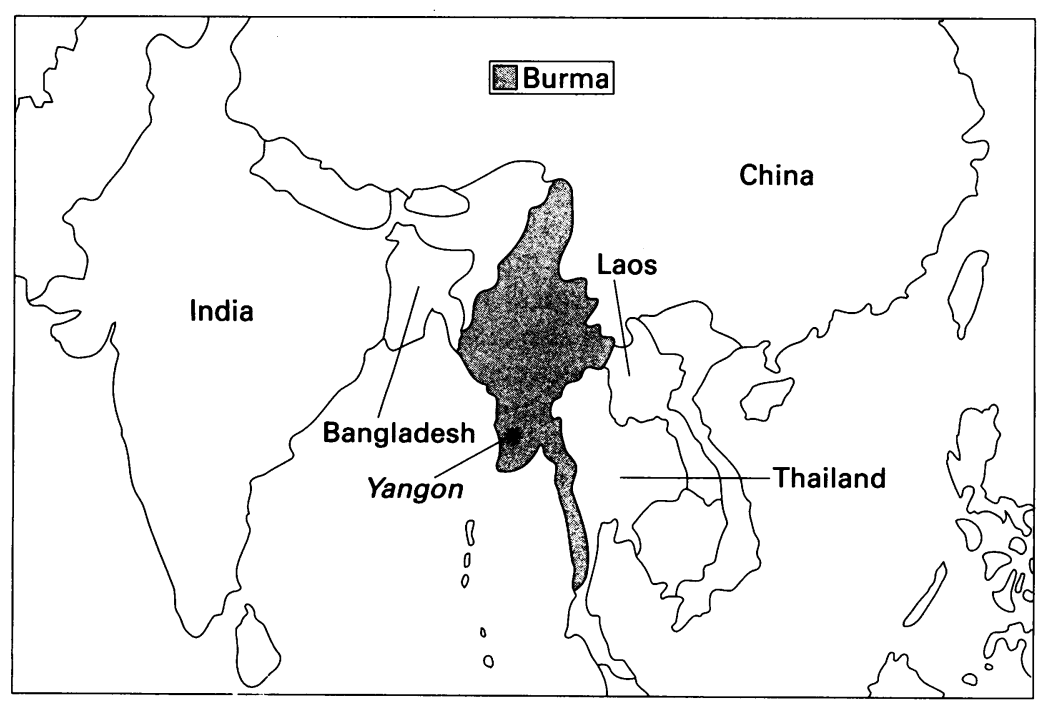

Figure 1 Map of Burma and surrounding countries. population of approximately 15 million in 82 townships (in 14 districts).

Efforts to prevent trachomatous visual impairment in Burma consist of health education and topical treatment of infection with antibiotics (herein referred to as non-surgical interventions) and surgical correction of trichiasis and entropion (herein referred to as surgical intervention).

\section{NON-SURGICAL INTERVENTIONS}

Community education and antibiotic treatment for trachoma infection have been and remain the principal constituents of 'nonsurgical' interventions. These activities which receive local political and media support are delivered by employees of the TCP in collaboration with a wide range of community workers such as school teachers and midwives. Eye health education is integrated into primary school curricula and reinforced through regular monitoring and treatment of trachoma infection of schoolchildren by the TCP. Antibiotic treatment with $1 \%$ topical tetracycline ointment is carried out in three successive stages: (1) the attack stage, whereby treatment is given to the entire population in areas of high endemicity $(30 \%$ or greater prevalence of active trachoma); (2) the consolidation stage, during which treatment courses are repeated whenever the prevalence of active trachoma is higher than $15 \%$; and (3) the maintenance stage, during which selective treatment of infection continues. By the end of the programme's first phase (1977-8), the attack stage had been completed in 45 townships ${ }^{3}$; thus, the emphasis in phases 2 and 3 was on consolidation and maintenance.

SURGICAL INTERVENTION

A modification of the 'grey line splitting' technique is used to surgically correct entropion and trichiasis in Burma. ${ }^{4}$ Operations, performed in the villages by trained auxiliary health personnel employed by the TCP, can be completed in 10 to 20 minutes. Following surgery, patients are instructed in appropriate wound hygiene and told to return to the local health clinic approximately 11 days later to have the sutures and plastic splint removed.

Representative samples of rural populations are randomly selected for eye examinations to monitor trachoma infection, potentially disabling, and disabling eye lesions before and after interventions ${ }^{3}$ thereby providing crucial feedback to the activities of the TCP. It was not until 1990, however, with the introduction of the annual model eye health survey (MEHS), that the prevalence of trachomatous blindness and low vision, where blindness is defined as visual acuity in the better eye of less than 3/60 and low vision as visual acuity in the better eye less than $6 / 18$ but greater than $3 / 60$, could be determined.

The 30 year period during which the programme has been operating can be divided into three distinct phases: phase 1 (1964/6577/78), characterised by vertical control of trachoma in highly endemic areas employing sustained attack stage treatments as described 
above, with subsequent dramatic decreases in the prevalence of trachoma infection and potentially disabling lesions ${ }^{3}$; phase 2 (1978/79$88 / 89$ ), characterised by consolidation and maintenance strategies for trachoma control (given the success of phase 1) and a horizontal integration of the TCP into basic health services whereby all blinding conditions in the trachoma endemic area became the concern of the programme; and phase 3 (1989/90-93/94), during which the responsibilities of the programme were expanded to include the control of all blinding conditions throughout Burma advocating the primary eye care approach by utilising basic health services and primary health care infrastructure.

\section{Methods}

costs

The analysis considers direct costs to the health care system only. Indirect costs-that is, those borne by patients or their families although recognised as important, are not included. In addition to the long term funding by the Burma Ministry of Health, additional resources have been provided by the World Health Organisation (1966 to the present), UNICEF (1967-89), United Nations Development Programme and a variety of nongovernmental organisations. Data on the costs of the Burma TCP were obtained primarily from files kept at the programme headquarters in Yangon, with additional information extracted from documents provided by the World Health Organisation in Geneva as well as the UNICEF and UNDP representatives in Yangon. Data were collected according to four broad categories: vehicles, personnel, buildings, and supplies and equipment. Each of these categories is further divided into capital and recurrent costs. Capital costs, items that are consumed over more than 1 year, include the training of personnel and the purchase of vehicles, buildings and supplies, and equipment. Recurrent costs include vehicle fuel and maintenance, salaries, building rental, maintenance and utilities, medicines, and consumable supplies and equipment. A number of cost items had no budget line-for example, buildings. These non-traded goods were valued according to current, local fair market value. Surgical and non-surgical interventions were provided to the population free of charge. GDP deflators and market exchange rates from the 1993 International financial statistics yearbook are used to convert all costs to 1990 US dollars. ${ }^{5}$ Capital costs are converted to an equivalent annual cost based on an estimated lifespan and an annual interest rate of $3 \%$.

Since the inception of the TCP, services for eye diseases other than just trachoma, including cataract surgery, have been extended to the population. The total costs of the TCP as determined above represented the joint cost of providing trachoma and other eye health interventions. The percentage of annual costs attributable to trachoma were determined by having the programme director estimate the percentage of costs from each of the four major categories which resulted from trachoma work alone. To incorporate the evolving nature of the programme over the 30 year period, the relative percentage of costs attributable to trachoma control were assessed for the three phases identified above. The apportioning exercise also was influenced by the nature of the costs in respective categories. For example, personnel apportioning was based on the percentage of time each different occupation category spent on trachoma control, weighted by the salary level and number of employees per occupation category. The resulting percentage of personnel time spent on trachoma control was used for other cost categories where similar precision was not available. A further apportioning exercise was conducted to divide trachoma control costs between nonsurgical and surgical interventions. These costs were further broken down according to whether they were fixed or variable-that is, independent or dependent of the numbers of people treated by the programme, respectively. In this analysis, marginal cost, or the cost of producing one more unit of an intervention, is assumed to be equal to average variable cost.

\section{EFFECTIVENESS}

Benefits arising from trachoma control are a function of the cases of trachomatous blindness prevented over the 30 year programme period. Only two prevalence surveys of trachomatous blindness deemed representative of the programme area could be identified; other existing surveys were rejected due to nonrandom sampling or insufficient details on survey methods. In 1965, the visual acuity and cause of visual impairment were determined for a $1 \%$ random sample $(n=21039)$ of people of all ages in four of the TCP districts before the commencement of the programme. ${ }^{6}$ The prevalence of blindness and low vision was age/ sex standardised using the 1965 population distribution for Burma $^{7}$ and the resulting values were entered into the Harvard Incidence Prevalence Model to derive incidence rates for blindness and low vision. ${ }^{8}$ Finally, a single incidence for males and females is expressed for the precontrol target population in 1965 .

Using data from the 1993 Model Village Eye Health Survey, which included 25249 people from 14 randomly selected townships in the programme area, male and female incidence of trachomatous blindness and low vision for the target population in 1993 is derived in exactly the same way. The change in incidence from 1965 to 1993 is assumed to decrease linearly and to be due entirely to the TCP (that is, both non-surgical and surgical interventions, see Fig 2). It is also assumed that townships joining the TCP between 1965 and 1993 had incidence rates of blindness and low vision identical to rates in the programme area at the time of their joining, and that the incidence of trachomatous visual impairment in each township would have remained constant in the absence of intervention. Therefore, cases of trachomatous visual impairment prevented by the programme are equal to incidence in the absence of control minus incidence with control multiplied by the target population. 
Table 1 Effectiveness of 'grey line splitting' lid rotation surgery for trichiasis/entropion for each phase of the trachoma control programme

\begin{tabular}{llllll}
\hline & $\begin{array}{l}\text { Efficacy of } \\
\text { trichiasis } \\
\text { surgery }\end{array}$ & $\begin{array}{l}\text { Average number of } \\
\text { surgeries performed per } \\
\text { year }\end{array}$ & $\begin{array}{l}\text { Average number of } \\
\text { trichiasis/entropion } \\
\text { cases }\end{array}$ & $\begin{array}{l}\text { Coveragelcompliance of } \\
\text { trichiasis surgery }\end{array}$ & $\begin{array}{l}\text { Effectiveness of } \\
\text { trichiasis surgery }\end{array}$ \\
\hline $1(1964 / 65-77)$ & $76 \%$ & 5295 & 114129 & $5 \%$ & $4 \%$ \\
$2(1978 / 79-88)$ & $76 \%$ & 4345 & 145925 & $3 \%$ & $2 \%$ \\
$3(1989 / 90-93)$ & $76 \%$ & 5187 & 140512 & $4 \%$ & $3 \%$ \\
$1964-5$ & $76 \%$ & 4929 & 35640 & $14 \%$ & $10 \%$ \\
\hline
\end{tabular}

The contribution of surgical intervention to the decrease in incidence is assumed to be a function of the effectiveness of the 'grey line splitting' procedure. Effectiveness of an intervention is determined by the following three factors: (1) efficacy; (2) patient and health care provider compliance; and (3) coverage of the population at risk. The efficacy of lid rotation surgery at preventing recurrence of entropion is assumed to be the efficacy of lid rotation surgery at preventing visual impairment. Because a more precise measure of calculating the efficacy of lid rotation surgery would require knowledge of both the percentage of people who maintain normal vision despite the recurrence of trichiasis/entropion as well as the percentage of people who go on to become visually impaired even though trichiasis/ entropion does nor recur (data that were not available), a value of $76 \%$ is selected based on
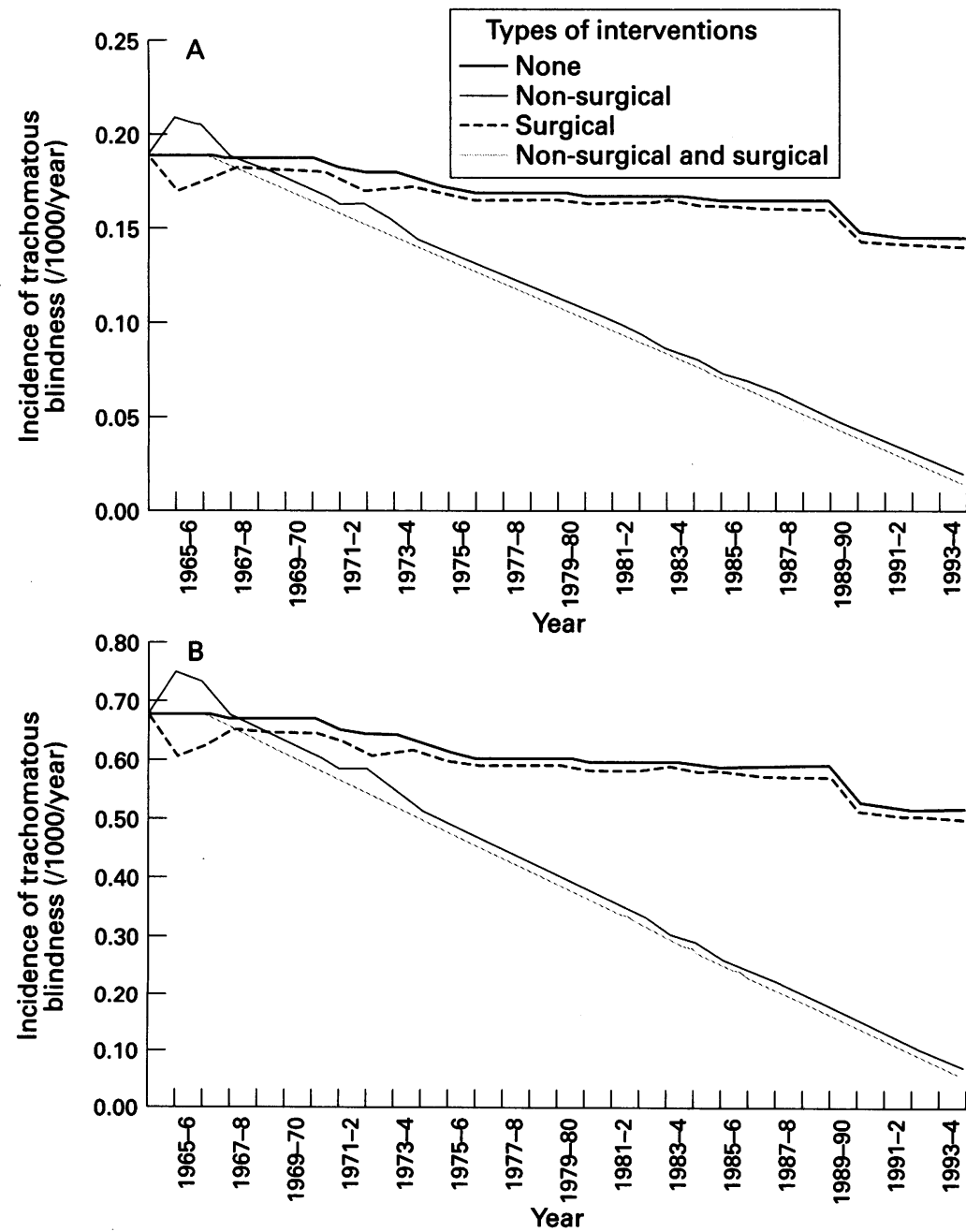

Figure 2 The incidence of trachomatous blindness in males $(A)$ and females $(B)$ with no intervention, non-surgical interventions only, surgical interventions only, or non-surgical and surgical interventions combined. a 1993 study in two districts of Burma which found that 811 of 1069 entropion/trichiasis cases had not recurred at an average time of 8.51 years following grey line splitting lid rotation surgery. Coverage and compliance are calculated by dividing the number of lid rotation surgeries performed per annum by the number of entropion/trichiasis cases in the trachoma control area (which is the product of the prevalence of entropion/trichiasis and target population). The gross prevalence of entropion/ trichiasis in the TCP area for 1965 and 1993 was derived from the aforementioned surveys. The resulting coverage/compliance figures when averaged during each phase of the programme range from $3 \%$ to $5 \%$ (Table 1 ). Multiplying coverage/compliance by the efficacy yields the effectiveness of lid rotation surgery at preventing trachomatous visual impairment, which averages between $2 \%$ and $4 \%$ depending on the programme phase (Table 1). The annual effectiveness is multiplied by the corresponding annual incidence to identify the incident cases of trachomatous visual impairment prevented by surgical intervention.

The incidence of cases of trachomatous visual impairment prevented by non-surgical interventions is the incidence prevented by surgical prevention subtracted from the incidence prevented by the programme as a whole. Multiplying the target population by the annual incidence prevented for each of the two control strategies identifies the annual cases of trachomatous blindness and low vision prevented by non-surgical and surgical interventions.

UTILITY

The burden of trachomatous visual impairment is measured using a composite indicator of health status that incorporates information on both mortality and handicap, called handicap adjusted life years (HALYs). HALYs, like other composite measures of ill health such as DALYs (disability adjusted life years) and QALYs (quality adjusted life years), expresses as a single unit the years of life lost due to premature mortality and life years lost while living with morbidity, disability, or handicap for each individual with a disease ${ }^{89}$ Handicap refers to the disadvantage experienced by the impaired individual - that is, a blind person may be unable to work or find a spouse. The degree of handicap or disadvantage is expressed as a weight ranging between 0 and 1 ; being completely handicapped (weight of 1) is equivalent to death while having no handicap (weight of 0 ) is equivalent to perfect health.

Years of healthy life lost from handicap is the 
Table 2 Means and lower and upper bound estimates for each of the six variables included in the sensitivity analysis

\begin{tabular}{|c|c|c|c|}
\hline Variable & Mean & Lower bound estimate & Upper bound estimate \\
\hline $\begin{array}{l}\text { (1) Discount rate: } \\
\text { Cost effectiveness of primary interventions (1990 US\$) } \\
\text { Cost effectiveness of secondary interventions (1990 US } \$ \text { ) }\end{array}$ & $3.0 \%$ & $\begin{array}{l}0.0 \% \\
7(63 \%) \\
44(74 \%)\end{array}$ & $\begin{array}{l}10.0 \% \\
33(285 \%) \\
104(177 \%)\end{array}$ \\
\hline \multicolumn{4}{|l|}{ (2) Prevalence of trachomatous blindness and low vision 1965: } \\
\hline Blindness & 4.71 & 2.36 & 7.07 \\
\hline Low vision & 14.15 & 7.08 & 21.23 \\
\hline \multicolumn{4}{|l|}{ (3) Prevalence of trachomatous blindness and low vision 1993: } \\
\hline Blindness & 0.39 & 0.19 & 0.58 \\
\hline Low vision & 1.16 & 0.58 & 1.74 \\
\hline \multicolumn{4}{|l|}{ (4) Increased risk of death: } \\
\hline Blind males & 2.5 & 1.3 & 3.8 \\
\hline Blind females & 3.8 & 1.9 & 5.7 \\
\hline Low vision males & 1.4 & 0.7 & 2.1 \\
\hline Low vision females & 1.5 & 0.8 & 2.3 \\
\hline \multicolumn{4}{|l|}{ (5) Handicap weights: } \\
\hline Blindness & 0.58 & 0.53 & 0.63 \\
\hline Low vision & 0.59 & 0.51 & 0.67 \\
\hline \multicolumn{4}{|l|}{ (6) Effectiveness of non-surgical and surgical interventions: } \\
\hline Non-surgical & $41.9 \%$ & $20.9 \%$ & $43.5 \%$ \\
\hline Surgical & $3.2 \%$ & $1.6 \%$ & $4.8 \%$ \\
\hline
\end{tabular}

product of population life expectancy of the visually impaired at a given age and the handicap weight-that is, a 45 -year-old blind man with a life expectancy of 20 years and a handicap weight of 0.5 would lose 10 years of healthy life due to handicap. Similarly, years of life lost as a result of premature mortality are calculated by multiplying the number of excess deaths among the visually impaired at a given age by the life expectancy at that age.

\section{PREMATURE MORTALITY}

The increased risk of mortality conferred by blindness and low vision is derived from data gathered by the Onchocerciasis Control Programme in west Africa where mortality was found to be 2.5 times higher among blind males and 3.8 times higher among blind females compared with sighted controls. ${ }^{10}$ Lower but still significantly elevated mortality risk was associated with low vision, at 1.4 for males and 1.5 for females. ${ }^{10}$ Although other studies have found a similar relation for trachoma blindness, ${ }^{11}$ the Kirkwood study is chosen based on its large sample size and the definitions of blindness and low vision employed.

YEARS LOST DUE TO PREMATURE MORTALITY

The number of years of life lost as a result of death at each age are obtained from family model life tables ${ }^{12}$ which provide age-specific standard expectations of life. Models with life expectancies of 80 and 82.5 years are used as the standards for males and females, respectively.

\section{YEARS LIVED WITH VISUAL IMPAIRMENT}

The number of years of life lived after becoming visually impaired at each age are obtained from standard life tables for the visually impaired. These tables are constructed by adjusting the reference tables to reflect the increased age-specific mortality conferred by trachomatous blindness and low vision. ${ }^{8}$

HANDICAP ADJUSTED LIFE YEARS

Although a number of studies have described the significant socioeconomic disadvantage experienced by the blind and low visioned, ${ }^{1314}$ there has been only one study in which an attempt was made to quantify the handicap of the visually impaired (Evans TG, Ranson MK, unpublished data, 1994). In the absence of any other empirical work from which such coefficients could be derived, these weights are selected recognising that they may not be representative of the visually impaired in Burma; further empirical work will most likely show them to be sensitive to age, sex, and region (among other things). Using a casecontrol design, 100 visually impaired ( 69 blind and 31 visually impaired) and 45 well sighted age/sex matched controls were interviewed in an area of hyperendemic trachoma in central Tanzania to identify the disadvantage experienced in each of four areas: mobility, social integration, work, and household role. A handicap scale was derived based on a single self assessment of disadvantage by the respondent within each of the four domains and overall. The mean handicap scale scores reveal similar levels of handicap among the blind and low visioned which are significantly higher than among the well sighted (Fig 3). On the basis of these results handicap weights of 0.58 for the blind and 0.59 for the low visioned are selected. Years lived with decreased visual acuity are adjusted by this handicap weight $(0$, representing perfect health and 1 , representing death) to give handicap adjusted life years.

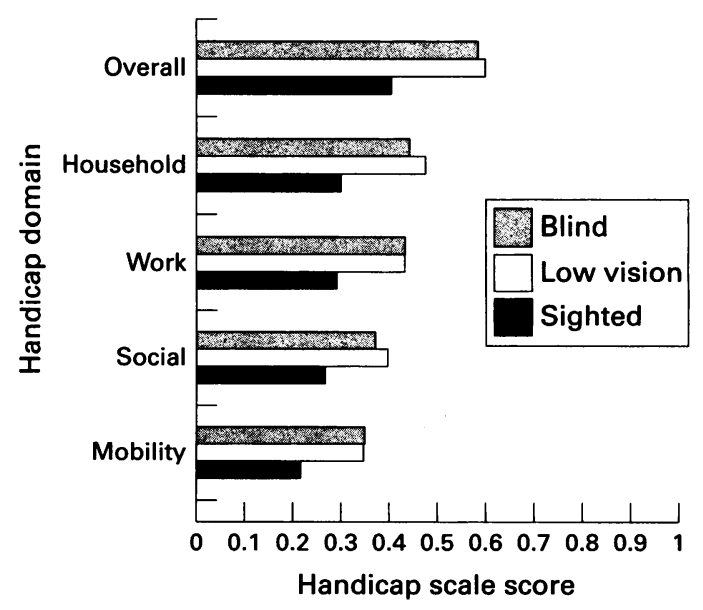

Figure 3 Handicap scale scores according to visual acuity. 
Table 3 Annual total cost and variable cost of non-surgical and surgical interventions for each phase of the trachoma control programme (1990 \$ per 100000 population)

\begin{tabular}{|c|c|c|c|c|c|c|}
\hline \multirow{2}{*}{ Phase } & \multicolumn{2}{|c|}{ Non-surgical interventions } & \multicolumn{2}{|c|}{ Surgical intervention } & \multicolumn{2}{|c|}{ Combined } \\
\hline & Total & Variable & Total & Variable & Total & Variable \\
\hline $\begin{array}{l}1(1964 / 65-77 / 78) \\
2(1978 / 79-88 / 89) \\
3(1989 / 90-93 / 94)\end{array}$ & $\begin{array}{r}18335 \\
9301 \\
1806\end{array}$ & $\begin{array}{r}4952 \\
3114 \\
285\end{array}$ & $\begin{array}{r}6945 \\
2806 \\
754\end{array}$ & $\begin{array}{r}1370 \\
410 \\
101\end{array}$ & $\begin{array}{r}25279 \\
12108 \\
2559\end{array}$ & $\begin{array}{r}6321 \\
3524 \\
386\end{array}$ \\
\hline Average & 9895 & 2875 & 3459 & 607 & 13354 & 3483 \\
\hline
\end{tabular}

DISCOUNTING FUTURE HALYS

To express future years of life lost or lived with handicap in the present, an annual discount rate or social time preference of $3 \%$ is selected.

\section{Cost effectiveness and cost utility}

The cost effectiveness of non-surgical and surgical interventions for trachomatous visual impairment is calculated for each of the programme's three phases and is expressed as cost (in 1990 US dollars) per case of visual impairment prevented. Likewise, the cost utility of primary non-surgical and surgical prevention is calculated for each phase of the programme and is expressed as cost per HALY saved.

\section{Sensitivity analysis}

To evaluate the dependence of the cost utility of non-surgical and surgical interventions on underlying assumptions, analyses are performed for a given range of values for each of the following six variables: the discount rate, the prevalence of trachomatous blindness and low vision in 1965 and in 1993, the increased risk of death associated with visual impairment, handicap weights for blindness and low vision, and the effectiveness of primary nonsurgical and surgical prevention (Table 2). For each of the variables, the value used in the calculation of cost effectiveness is varied over the range of uncertainty from a lower bound to a higher bound - for example, the discount rate is varied from $0 \%$ to $10 \%$.

\section{Results}

costs

Table 3 presents the average annual total and variable cost of non-surgical and surgical interventions for each phase of the programme. For both interventions, the annual cost is greatest during phase 1 , less expensive in phase 2 , and dramatically lower in phase 3 . Non-surgical interventions consume considerably greater resources than surgical resources in each phase, the mean annual total costs per 100000 population being $\$ 9895$ and $\$ 3459$ respectively. The corresponding total costs of 30 years of non-surgical and surgical interventions for trachomatous visual impairment in Burma are estimated to be $\$ 25.6 \mathrm{~m}$ and $\$ 9.0 \mathrm{~m}$, respectively. Breakdown of average annual costs shows that most of the money spent on non-surgical interventions is accounted for by the costs of training and paying personnel, followed by costs of supplies and equipment, vehicle costs, and costs of buildings (Fig 4A). The distribution of money that has been spent on surgical prevention is similar with a slightly larger portion accounted for by personnel (Fig 4B).

PROGRAMME EFFECTIVENESS AND UTILITY

The number of new cases of visual impairment in the absence of trachoma control minus the number of cases with the control yields the programme effectiveness-that is, cases of visual impairment prevented by the TCP. The average annual programme effectiveness of both non-surgical and surgical interventions for each phase of the TCP is presented in Table 4. The increasing effectiveness over time of non-surgical interventions (Fig 2) is reflected by the 91 cases of visual impairment prevented per 100000 population annually during phase 3 , compared with only 69 during phase 2 and 21 during phase 1 . Likewise, the constant or slightly decreasing effectiveness of surgical
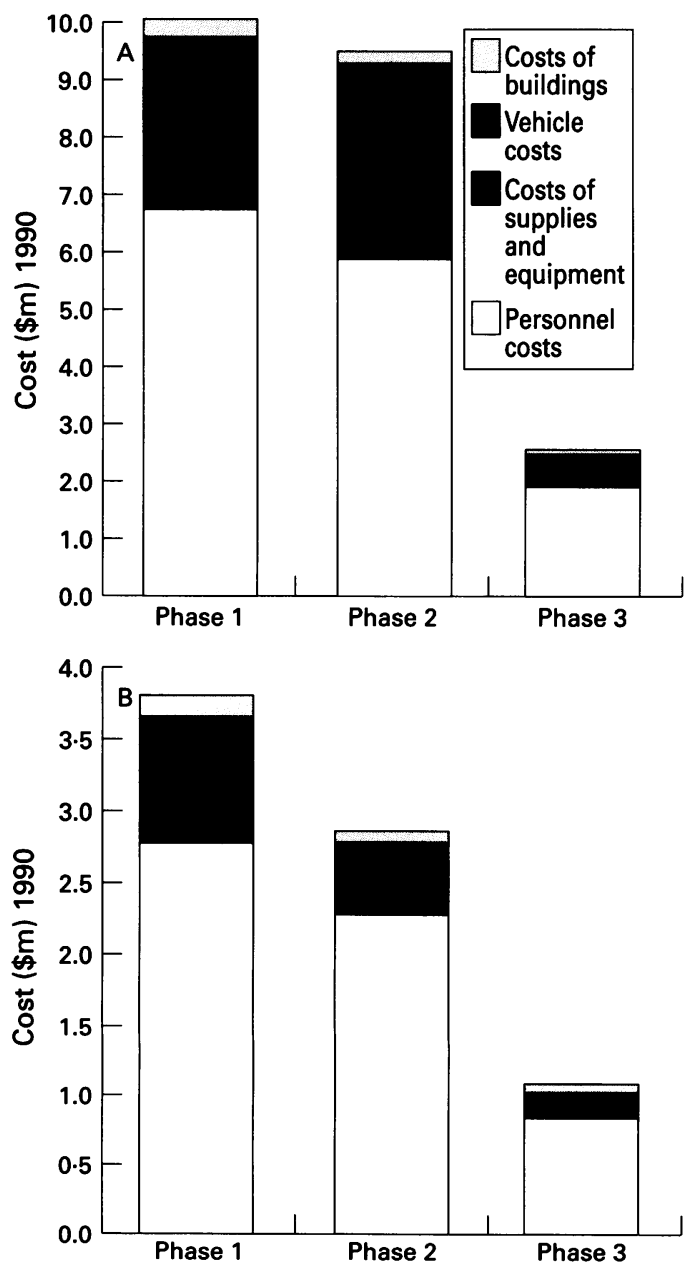

Figure 4 Breakdown of average annual costs of non-surgical $(A)$ and surgical $(B)$ interventions for trachomatous visual impairment for each phase of the trachoma control programme. 
Table 4 Average annual programme effectiveness of non-surgical and surgical interventions for each phase of the trachoma control programme: cases of visual impairment prevented per 100000 population

\begin{tabular}{llll}
\hline Phase & Non-surgical & Surgical & Combined \\
\hline $1(1964 / 65-77 / 78)$ & 21 & 5 & 26 \\
$2(1978 / 79-88 / 89)$ & 69 & 3 & 71 \\
$3(1989 / 90-93 / 94)$ & 91 & 2 & 94 \\
Average & 61 & 3 & 64 \\
\hline
\end{tabular}

Table 5 Average annual utility of non-surgical and surgical interventions for each phase of the trachoma control programme: HALYs saved per 100000 population

\begin{tabular}{lclc}
\hline Phase & Non-surgical & Surgical & Combined \\
\hline $1(1964 / 65-77 / 78)$ & 372 & 99 & 471 \\
$2(1978 / 79-88 / 89)$ & 1018 & 46 & 1064 \\
$3(1989 / 90-93 / 94)$ & 1144 & 35 & 1179 \\
Average & 862 & 59 & 921 \\
\hline
\end{tabular}

interventions (Table 1) underlies the relatively constant programme effectiveness of between two to five cases of visual impairment prevented per 100000 population annually for each phase of the TCP. Finally, on the basis of the overall average annual programme effectiveness, it is estimated that more than 200000 cases of visual impairment have been prevented by non-surgical interventions while fewer than 9000 cases have been prevented by surgical interventions over the 30 year history of the TCP.

By adding the years of healthy life gained and years lived without handicap associated with each prevented case of visual impairment, the utility (measured in HALYs) arising from programme effectiveness is derived. The average annual utility of non-surgical and surgical interventions per 100000 population is 862 and 59 HALYs respectively (Table 5 ). Considered over the 30 year programme period, a total of almost 3 million HALYs have been saved solely by non-surgical interventions while only one-eighteenth of this amount (170 000 HALYs) has been saved by surgical intervention alone. For the same reasons as described for programme effectiveness above,

Table 6 The average and minimal cost effectiveness of non-surgical and surgical interventions for each phase of the trachoma control programme: 1990 \$/case of visual impairment prevented

\begin{tabular}{|c|c|c|c|c|c|c|}
\hline \multirow{2}{*}{ Phase } & \multicolumn{2}{|c|}{ Non-surgical } & \multicolumn{2}{|l|}{ Surgical } & \multicolumn{2}{|c|}{ Combined } \\
\hline & Average & Marginal & Average & Marginal & Average & Marginal \\
\hline $\begin{array}{l}1(1964 / 65-77 / 78) \\
2(1978 / 79-88 / 89) \\
3(1989 / 90-93 / 94)\end{array}$ & $\begin{array}{r}876 \\
136 \\
20\end{array}$ & $\begin{array}{r}237 \\
45 \\
3\end{array}$ & $\begin{array}{r}1489 \\
1100 \\
306\end{array}$ & $\begin{array}{r}294 \\
161 \\
41\end{array}$ & $\begin{array}{r}988 \\
170 \\
27\end{array}$ & $\begin{array}{r}247 \\
50 \\
4\end{array}$ \\
\hline Average & 163 & 47 & 1099 & 193 & 209 & 54 \\
\hline
\end{tabular}

Table 7 The average and minimal cost utility of non-surgical and surgical interventions for each phase of the trachoma control programme: 1990 \$/HALY saved

\begin{tabular}{|c|c|c|c|c|c|c|}
\hline \multirow{2}{*}{ Phase } & \multicolumn{2}{|c|}{ Non-surgical } & \multicolumn{2}{|l|}{ Surgical } & \multicolumn{2}{|c|}{ Combined } \\
\hline & Average & Marginal & Average & Marginal & Average & Marginal \\
\hline $\begin{array}{l}1(1964 / 65-77 / 78) \\
2(1978 / 79-88 / 89) \\
3(1989 / 90-93 / 94)\end{array}$ & $\begin{array}{r}49 \\
9 \\
2\end{array}$ & $\begin{array}{r}13 \\
3 \\
0\end{array}$ & $\begin{array}{l}70 \\
60 \\
22\end{array}$ & $\begin{array}{r}14 \\
9 \\
3\end{array}$ & $\begin{array}{r}54 \\
11 \\
2\end{array}$ & $\begin{array}{r}13 \\
3 \\
0\end{array}$ \\
\hline Average & 11 & 3 & 59 & 10 & 14 & 4 \\
\hline
\end{tabular}

there is a dramatic increase in HALYs saved in each successive programme phase for nonsurgical interventions whereas utility is more or less constant in each phase of the TCP for surgical interventions.

COST EFFECTIVENESS AND COST UTILITY

The total annual average cost effectiveness of non-surgical interventions over the 30 year duration of the TCP is found to be $\$ 163$ per case of visual impairment prevented (Table 6 ). Surgical prevention is almost six times less cost effective at $\$ 1099$ per case of visual impairment prevented. The average cost effectiveness of both types of prevention improved dramatically over time; non-surgical interventions were 44 times more cost effective and surgical intervention five times more cost effective during phase 3 than phase 1 . A similar improvement in the marginal cost effectiveness is seen with each progressive phase, the average annual marginal cost effectiveness being $\$ 47$ and $\$ 193$ per case of visual impairment prevented for non-surgical and surgical interventions respectively. For the programme as a whole the marginal cost of preventing a case of visual impairment is $\$ 54$ with an average cost of $\$ 209$.

The total annual average cost utility of nonsurgical interventions is found to be about $\$ 11$ per HALY saved compared with $\$ 59$ per HALY saved for surgical intervention (Table 7). This difference between non-surgical and surgical is diminished when comparing marginal cost utility: $\$ 3$ and $\$ 10$ per HALY saved respectively. For both non-surgical and surgical interventions a dramatic improvement in cost utility is observed from phase 1 through to phase 3 . The combined average and marginal cost utilities are $\$ 14$ per HALY and $\$ 4$ per HALY respectively.

\section{SENSITIVITY ANALYSIS}

Figure 5 illustrates the lowest and highest values of the cost utility of non-surgical and surgical interventions generated for each of the six variables included in the sensitivity analysis. Under the 'high HALYs' assumption, the cost utility improves (that is, the $\$ / \mathrm{HALY}$ ratio is lower), whereas, the 'low HALYs' assumption worsens the cost utility (that is, the $\$ / \mathrm{HALY}$ ratio is higher). The cost utility values of both types of prevention are relatively insensitive to variation in the prevalence of trachomatous blindness and low vision in 1993, handicap weights, and the increased risk of death conferred by visual impairment. In contrast, the cost utility of both non-surgical and surgical interventions is very sensitive to the discount rate and the 1965 prevalence of blindness: a higher discount rate and a lower prevalence of blindness at the commencement of the TCP would substantially diminish the cost utility of these interventions. Remarkably, the cost utility of non-surgical interventions is highly sensitive to a small absolute change in the effectiveness of trichiasis surgery: increasing the effectiveness from $3.2 \%$ to $4.8 \%$ lowers the $\$ /$ HALY ratio to $67 \%$ of the mean corresponding to a $33 \%$ improvement in average cost utility. A $50 \%$ decline in the effective- 
ness of non-surgical interventions leads to a doubling of the average cost utility from $\$ 11.47$ per HALY to $\$ 22.94$ per HALY. Given the model for the decline in the prevalence of blindness adopted in this analysis, non-surgical interventions can only become marginally more effective.

\section{Discussion}

Perhaps the most striking impression emerging from this analysis of 30 years of trachoma control in Burma is the extraordinary decline in the incidence of trachomatous visual impairment. Earlier published analyses which identified dramatic decreases in the prevalence of trachoma infection and potentially disabling lesions, ${ }^{3}$ appear to have been accompanied by a decline in incidence of blindness and low vision. This analysis assumes that $100 \%$ of this decrease in incidence is attributable to the TCP, mostly through non-surgical interventions-that is, community based education, antibiotic treatment of active trachoma, and surveillance of trachoma infection. The assumptions in this analysis, therefore, tend to provide a maximal, or generous, estimate of the effectiveness of non-surgical interventions and

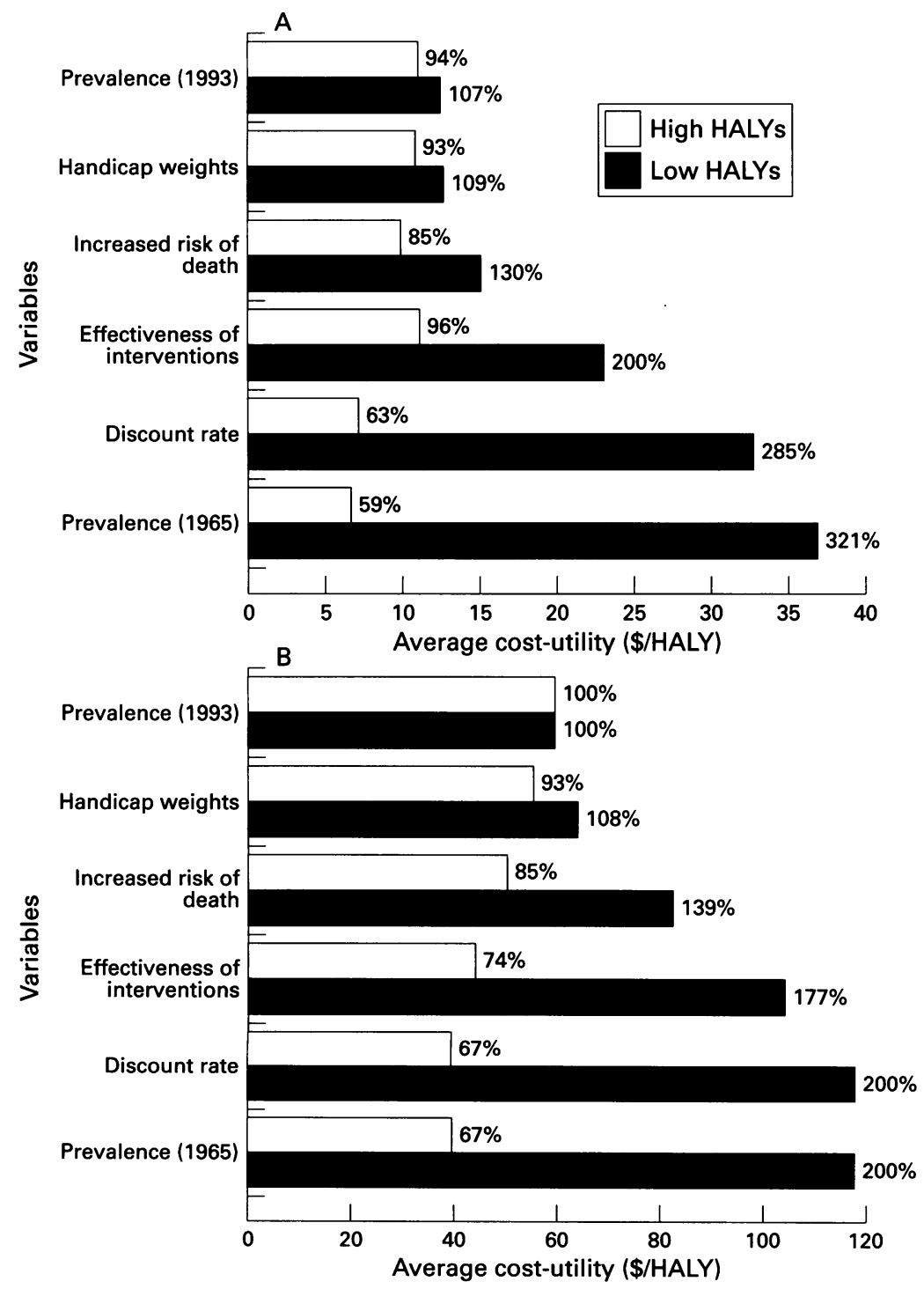

Figure 5 Sensitivity of cost utility results for non-surgical $(A)$ and surgical (B) interventions according to changes in six variables. a minimal, or conservative, estimate of the effectiveness of trichiasis surgery.

It is recognised that general levels of socioeconomic development have a strong association with trachomatous visual impairment, suggesting that in Burma part of the decline in trachoma endemicity and visual impairment may have been due to improvements in standards of living independent of the TCP. No formal attempt to test this hypothesis is made here, although informally it is acknowledged that the formerly trachoma endemic zone is characterised by low literacy, high infant mortality, poor water supply, and the absence of major natural resources relative to other areas of Burma. Furthermore, much of Burma's economic history in the past 30 years has been marked by systemic inefficiencies, deteriorating infrastructures, and commercial stagnation. On the surface, therefore, it seems that the dramatic change in the prevalence of trachomatous visual impairment in Burma over the past 30 years is less likely to be explained by secular changes in standards of living and supports the hypothesis put forward by West and Taylor' 15 that 'controlling trachomatous blindness need not await major socioeconomic changes in endemic countries'.

The assumption that community education and antibiotic treatment have been largely responsible for the decline appears to be at odds with the literature. Many of the studies evaluating the use of topical (or systemic) antibiotic treatment have documented recurrence of infection shortly after treatment leading authors to conclude that sustained treatment strategies are necessary to prevent the complications of trachoma infections. However, the sustainability of antibiotic treatment is generally thought to have little chance of long term success given problems with treatment compliance and costs. ${ }^{1617}$ The TCP in Burma appears to challenge this view and in this respect merits further in depth study. In particular, the nature of the decline in the incidence of visual impairment following the initiation of mass antibiotic treatment and education (that is, the slope of the line in Fig 2) largely determines the cost effectiveness of non-surgical interventions: if there is a long lag period, say 20 years, before a case of blindness is prevented, followed by a rapid decline in incidence, then non-surgical interventions would appear much less cost effective.

Measuring the effectiveness of surgical interventions is less problematic given data on efficacy and coverage. As identified from studies within the TCP, the grey line splitting technique has an efficacy of between $70 \%$ and $80 \%$; however, coverage appears to have been very low, thereby diminishing the potential effectiveness of surgical interventions. It is worth considering, however, that the prevalence of trichiasis may be overestimated and surgical coverage underestimated. Assuming the decrease in the prevalence of trachomatous blindness over 30 years is correct, a similar or slightly less pronounced decrease in trichiasis prevalence would be expected. ${ }^{17}$ As shown in Figure 6 , the decline in prevalence of tra- 


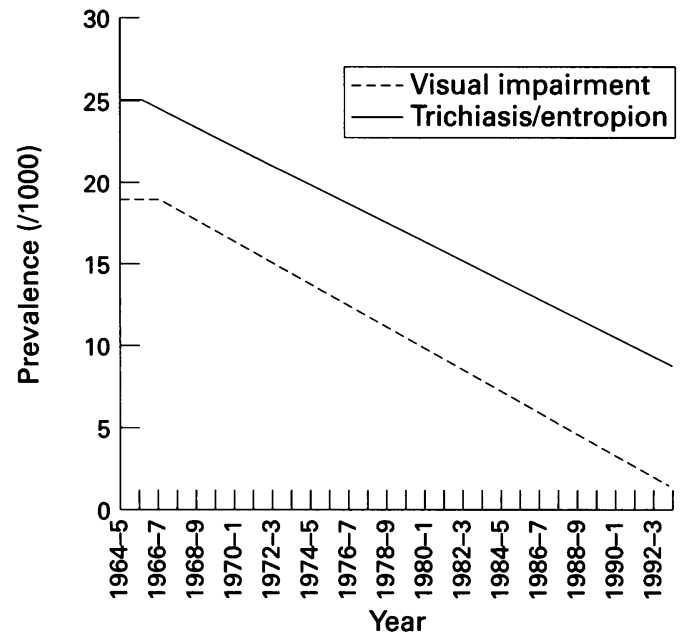

Figure 6 Change in the prevalence of trachomatous visual impairment and trichiasis/entropion from 1965 to 1993 in the trachoma control programme area.

chomatous blindness has a similar slope to the decline in trichiasis prevalence, suggesting that the prevalence of trichiasis is not likely to be overestimated. Accepting that the trichiasis prevalence figures are realistic, part of the explanation for low coverage may simply be a lack of awareness. It was only with the introduction of the MVEHS in 1991 that reliable trichiasis prevalence figures could be generated and hence surgical coverage assessed. Prior knowledge of low surgical coverage may have permitted a reallocation of priorities and resources to lid rotation surgery thereby increasing its effectiveness both in absolute terms and relative to non-surgical interventions. As demonstrated in this analysis, expanding the number of trichiasis surgeries performed is an attractive option given the low marginal cost effectiveness.

The experience of the TCP in Burma may also be instructional to other countries contemplating trachoma control initiatives. The programme has evolved through three distinct phases: phase 1 (1964/65-77/78) was characterised by concentrated attack stage, vertical control efforts to combat the high prevalence of trachoma infection and trachomatous visual impairment; phase $2(1978 / 79-88 / 89)$ by a decrease in the prevalence of trachoma infection and its complications and a shift to consolidation and maintenance activities provided in combination with other basic health services; and phase 3 (1989/90-93/94) by continued maintenance of trachoma control and negligible risk of trachomatous visual impairment. Not surprisingly, the resources devoted to trachoma control activities have diminished and the number of cases of trachomatous visual impairment prevented have increased with each phase; hence, the programme has become progressively more cost effective.

Rather than illustrating the relative advantages/disadvantages of vertical versus horizontal control, the Burma example offers more profound insight into how trachoma control programmes in other countries might be organised. It could be argued that a vertical attack stage, resource intensive programme was required in 1964 given the severity of endemic trachoma in the dry zone of Burma. An integrated, horizontal effort at that time, with limitations in the availability of personnel and material resources, may not have been capable of mounting sufficient control efforts to overcome endemic trachoma infection. Conversely, during phase 2 , continued vertical efforts may have been wasted given the dramatic improvement in trachoma endemicity. In deciding on the nature of a trachoma control programme, therefore, high endemicity areas may require vertical efforts initially whereas moderate or low endemicity regions might be more effectively served by an integrated, horizontal control strategy from the start. This experience of the TCP in Burma lends support to the therapeutic strategies for trachoma control as outlined by the World Health Organisation. ${ }^{18}$

Throughout the preceding analysis, nonsurgical and surgical interventions were compared, with a greater cost effectiveness and cost utility associated with the non-surgical interventions. As discussed above, there are reasons to believe that the effectiveness of non-surgical interventions may be overestimated and surgical effectiveness underestimated. Furthermore, the sensitivity of the cost effectiveness and cost utility measures to underlying assumptions suggests differences between the two interventions may be even smaller than the numbers suggest, especially when making comparisons at the margin. For example, if the lower bound of the effectiveness for antibiotics is used-that is, $20.9 \%$, then in order for the TCP to save the same number of HALYs, the effectiveness of the surgical interventions would have to increase to $24.2 \%$-assuming the efficacy of surgery is fixed at $76 \%$ this would require coverage to increase to about $32 \%$ of the trichiasis population, certainly not an unreasonable objective. If this were the case then the average cost effectiveness of surgery would be about $\$ 6.79 /$ HALY saved compared with $\$ 22.94$ for non-surgical interventions. Finally, comparing cost effectiveness between interventions, or across the health sector, is fraught with methodological difficulties and has led to the recommendation that order of magnitude dollar per benefit comparisons are programme

\begin{tabular}{llll}
\hline Phase & Number of surgeries performed & $\begin{array}{l}\text { Total cost per surgery performed } \\
(1990 \text { \$) }\end{array}$ & $\begin{array}{l}\text { Marginal cost per surgery performed } \\
(1990 \text { \$) }\end{array}$ \\
\hline $1(1964 / 65-77 / 78)$ & 74134 & 71.45 & 14.09 \\
$2(1978 / 79-88 / 89)$ & 47794 & 65.43 & 9.56 \\
$3(1989 / 90-93 / 94)$ & 25936 & 20.75 & 2.78 \\
Total & 147864 & 60.61 & 10.64 \\
\hline
\end{tabular}


more realistic than simply identifying whether an intervention is more or less cost effective. ${ }^{19}$ In this respect, the average and marginal cost utilities of surgical and non-surgical interventions differ by less than one order of magnitude. This implies that the relative differences in cost effectiveness and cost utility are not sufficient to suggest either non-surgical or surgical intervention is preferable to the other. This finding also supports policy recommendations for national trachoma control programmes which include both surgical and non-surgical strategies. $^{20}$

The subsequent question that arises is how the cost effectiveness of trachoma control compares with other blinding and nonblinding conditions. The limited literature on the cost effectiveness of control measures for blinding diseases and the absence of studies that have used HALYs as a measure of effectiveness limits these comparisons. Javitt, ${ }^{21}$ for example, estimates the marginal cost of cataract surgery to be between $\$ 18$ and $\$ 42$. In Table 8, the marginal cost per trichiasis surgery performed in Burma is between $\$ 2$ and $\$ 15$. Assuming the benefits from cataract and trichiasis surgery are similar in that they restore/preserve vision among the elderly, it is reasonable to assume that their cost effectiveness and utility are similar. Javitt identifies a range of $\$ 15$ to $\$ 27$ per DALY (disability adjusted life year) for cataract surgery. At this level of cost effectiveness, trichiasis surgery is among the most cost effective prevention activity. ${ }^{22}$ From our analysis, it follows that nonsurgical interventions, including community education and antibiotic treatment, also fit into this category.

The authors are grateful to the staff of the Trachoma Control Programme in Burma for their help in assembling information for this analysis. Further thanks are extended to the UNDP and UNICEF representatives in Yangon for availing their information. Comments were gratefully received from Dr Pararajasmation. Comments were gratefully received from Dr Pararajas-
egaram. Any errors or omissions remain the entire responsibil- ity of the authors. The study received financial support from the Edna McConnell Clark Foundation.

Sullivan LJ, Taylor HR. Trachoma. Sem Ophthalmol 1993;8:196-203.

2 Ko-Lay. Causes of blindness in Burma. Union of Burma fournal of Life Sciences 1968;1:85-7.

3 Tun Aung Kyaw, Thein Nyunt, Sundaresan TK, Tarizzo ML. Control of trachoma and prevention of blindness in rural communities in Burma. Bull World Hlth Organ 1978; 56:945-55.

4 Ba Hein, Aung Myint, Thein Dan. Follow up of entropion cases corrected by grey-line splitting operation in Sagaing township (Burmese). Rev Int Trach Pathol Ocul Trop Subtrop 1981;1-2:61-78.

5 International Monetary Fund. International financial statistics yearbook. New York: IFS, 1993.

$6 \mathrm{Ko} \mathrm{Ko,} \mathrm{Tun} \mathrm{Aung} \mathrm{Kyaw.} \mathrm{Socio-economic} \mathrm{factors} \mathrm{in}$ trachoma. Union of Burma fournal of Life Sciences 1968;1:365-9.

7 United Nations. The sex and age distributions of population. New York: UN, 1990.

8 Murray CJL. Quantifying the burden of disease: the technical basis for disability adjusted life years. Bull World Hith Organ 1994;72:429-45.

9 Drummond MF, Stoddard GL, Torrance GW. Methods for the economic evaluation of health care programmes. New York: Oxford Medical Publications, 1987.

10 Kirkwood B, Smith P, Marshall T, Prost A. Relationships between mortality, visual acuity and microfilarial load in the area of the onchocerciasis control programme. Trans Roy Soc Trop Med Hyg 1983;77:862-8.

11 Taylor HR, Katala S, Munoz B, Turner V. Increase in mortality associated with blindness in rural Africa. Bull World Hith Organ 1989;69:335-8.

12 Coale AJ, Guo G. Revised regional model life tables at very low levels of mortality. Population Index 1989; 55:613-643.

13 Evans TG. The socio-economic consequences of blinding onchocerciasis in West Africa. Bull World Hlth Organ 1995; 73:495-506.

14 Evans TG. The impact of permanent disability on rural households: river blindness in Guinea. IDS Bulletin 1989;20:41-8

15 West S, Taylor HR. Community-based intervention programs for trachoma control. Int Ophthalmol 1988;12:1923.

16 Bailey RL, Arullendran P, Whittle HC, Mabey DCW. Randomised controlled trial of single-dose azithromycin in treatment of trachoma. Lancet 1993;342:453-6.

17 Dawson CR, Schachter J. Strategies for treatment and control of blinding trachoma: costeffectiveness of topical or trol of blinding trachoma: costeffectiveness of topic

18 Dawson CR, Jones BR, Tarizzo ML. Guide to trachoma conDawson CR, Jones BR, Tarizzo ML. Guide to trach

19 World Bank. World development report 1993: investing in health. New York: Oxford University Press, 1993.

20 Thylefors B. Development of trachoma control programs and the involvement of national resources. Rev Infect Dis $1985 ; 7: 774-6$

21 Javitt JC. Cataract. In: Jamison DT, ed. Disease control priorities in developing countries. New York: Oxford University Press, 1993:635-46.

22 Jamison DT. Disease control priorities in developing countries: an overview. In: Jamison DT, ed. Disease control priorities in developing countries. New York: Oxford University Press, 1993:3-34. 